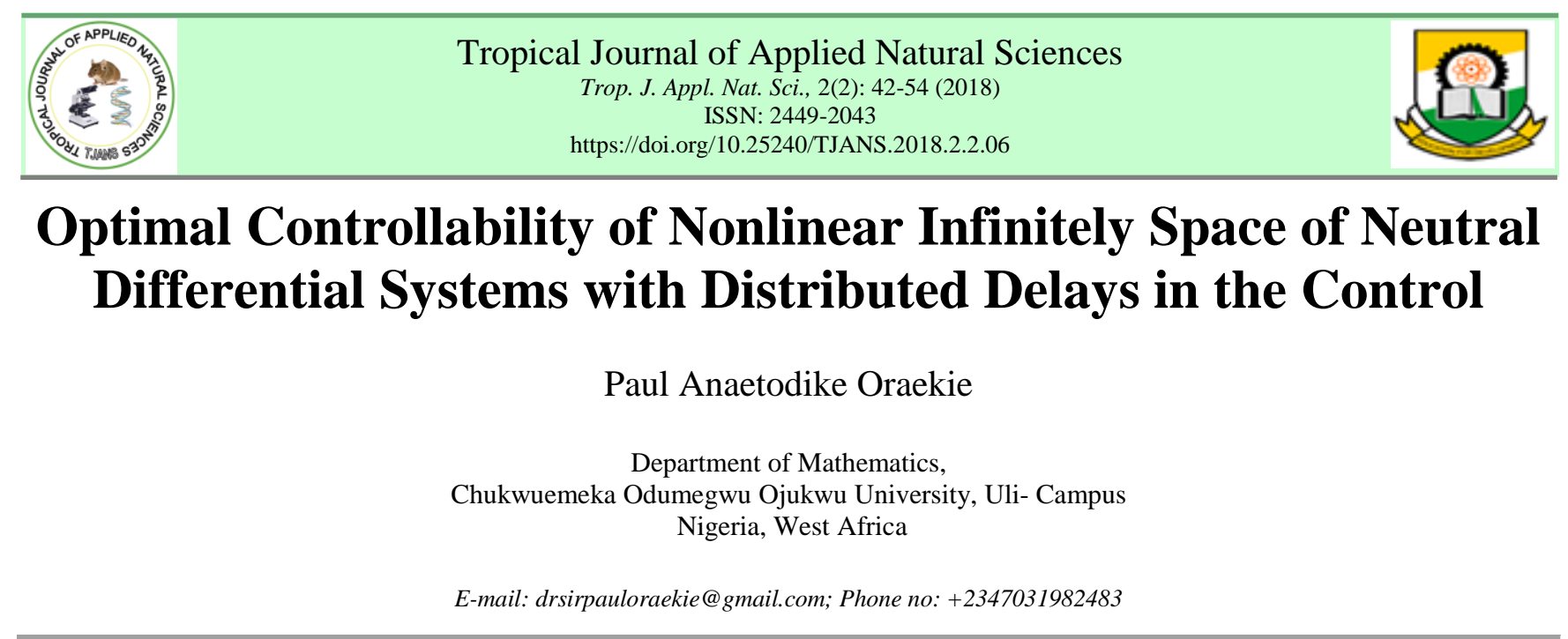

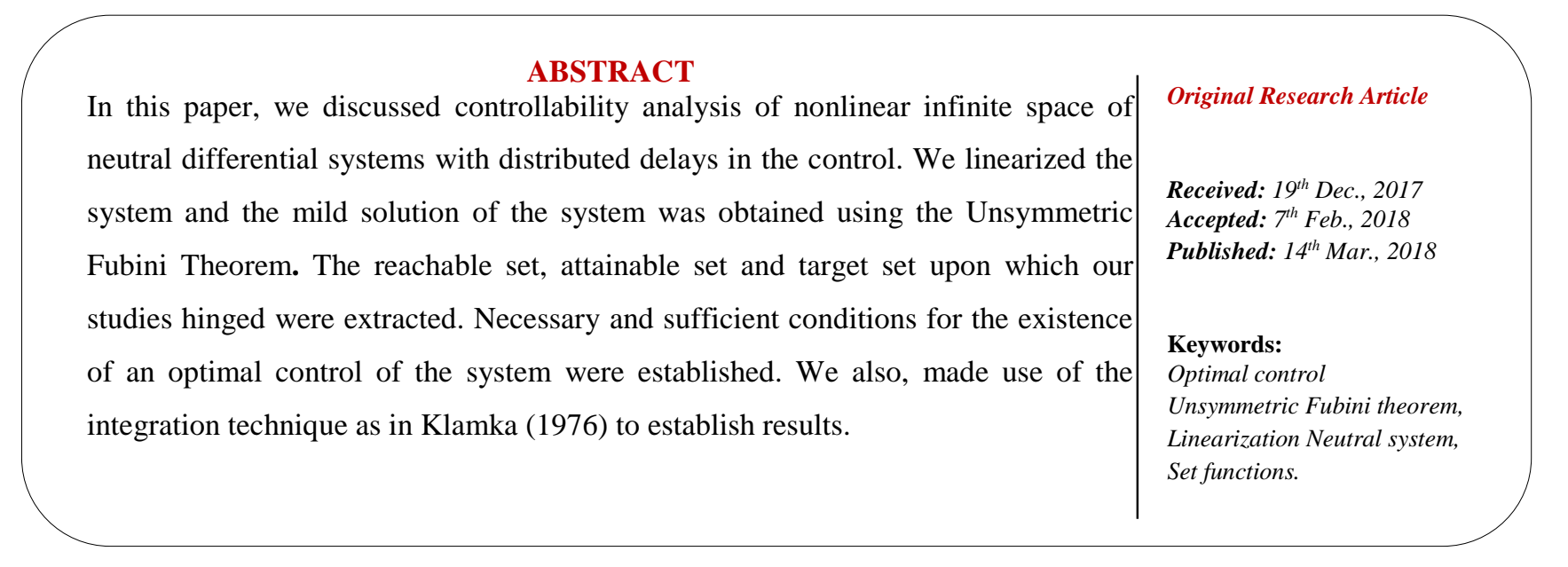

\title{
1. Introduction and Preliminaries
}

7 here are several papers which appeared on the controllability of nonlinear systems in infinite dimensional spaces. Balachandran and Anandhi (2003), discussed the controllability of neutral functional integro-differential systems in abstract phase space, with the help of Schauder fixed point theorem. Fu (2004), studied the same problem in abstract phase space for neutral functional differential systems with unbounded delay by using the Sado vskii fixed point theorem.

Onwuatu (1984), discussed the problem for nonlinear systems of neutral functional differential equations with limited controls.

However, the systems with delays in the both state and control, investigation into their relative controllability are still attracting attention and interest.

Optimality conditions for the relative controllability of nonlinear infinitely space of Neutral differential systems with distributed delays in the control is yet to be reported, though there are studies in the optimal controllability of ordinary and functional differential systems.

From the following studies, Artstein and Tradma (1982), Balachandran and Dauer (1989), Chukwu (2001), we gain clarity of the meaning and full understanding of the conceptual frame work of optimal controllability. 
In this work, we shall consider the Optimal Controllability of Nonlinear Infinitely Space of Neutral Differential Systems with Distributed Delays in the Control of the form:

$$
\frac{d}{d t}\left[D\left(t, x_{t}\right)\right]=L\left(t, x_{t}\right) x_{t}+\int_{-\infty}^{0} A(t, \theta) x(t+\theta) d \theta+\int_{-h}^{0} d_{\theta} H(t, \theta) u(t+\theta)+f\left(t, x_{t}\right) \ldots \ldots
$$

$x_{t_{0}}=\phi$

With the main objective 'to investigate' 'the existence of an optimal control of the system (1.1).

Let $n$ be a positive integer and $E=(-\infty, \infty)$ be the real line.

Denote by $E^{n}$ the space of real $n$ - tuples called the Euclidean space with norm denoted by|.|.

If $J=\left[t_{0}, t_{1}\right]$ is any interval of $E, L_{2}$ is the Lebesque space of square integrable functions from $J$ to $E^{n}$ written in full as $L_{2}\left(\left[t_{0}, t_{1}\right], E^{n}\right)$.

Let $h>0$ be positive real number and let $C\left([-h, 0], E^{n}\right)$ be the of Banach space of continuous functions with the norm of uniform convergence defined by

$$
\|\phi\|=\sup \phi(s) ;[-h, 0], \phi \in C\left([-h, 0], E^{n}\right)
$$

If $x$ is a function from $[-h, \infty]$ to $E^{n}$, then $x_{t}$ is a function defined on the delay interval $[-h, 0]$ given as

$$
x_{t}(s)=x(t+s) ; s \in[-h, 0], t \in[0, \infty) .
$$

Consider the nonlinear infinite neutral system

$$
\begin{aligned}
\frac{d}{d t}\left[D\left(t, x_{t}\right)\right]=L\left(t, x_{t}\right) x_{t} & +\int_{0}^{\infty} A(t, \theta) x(t+\theta) d \theta \\
& +\int_{-h}^{0}\left[d_{\theta} H(t, \theta)\right] u(t+\theta)+f\left(t, x_{t}\right) \ldots \ldots
\end{aligned}
$$

(Circularity of the function from $-\infty$ to 0 and from 0 to $\infty$ ).

Where

$$
\begin{gathered}
L\left(t, x_{t}\right)=\sum_{k=0}^{\infty} A_{k} x\left(t-w_{k}\right)+\int_{-\infty}^{0} A(t, \theta) x(t+\theta) d \theta \\
L\left(t, x_{t}\right) x_{t}=\int_{-h}^{0} d_{\theta} \mathrm{\eta}(t, s, x(t+s)) x(t+\theta) \\
\eta(t, s, \phi, \psi) \geq 0, \text { for } s \geq 0 \text { and } \phi, \psi \in C . \\
\eta(t, s, \phi, \psi) \text { exists for } t<-h
\end{gathered}
$$

$\mathrm{\eta}(t, s, \phi, \psi)$ is a continuous matrix function of bounded variation in $s ; s \in[-h, 0]$, (see Onwuatu, (1993))

$$
\operatorname{vary} \eta(t) \leq m(t), \quad m(t) \in L_{1}
$$


$L_{1}$ is the space of integrable functions.

Let $\Omega$ be an open subset of ExC and $D, L$ be bounded linear operators defined on ExC into $E^{n}$

$$
\begin{gathered}
\left|L\left(t, x_{t}\right) x_{t}\right| \leq m(t)\left\|x_{t}\right\|, \quad \text { for all } t \in E, \psi(t) \in C \\
D\left(t, x_{t}\right)=x(t) g\left(t, x_{t}\right),
\end{gathered}
$$

Where $g\left(t, x_{t}\right)=\sum_{n-o}^{\infty} A_{n}(t) \phi\left(t-w_{n}(t)+\int_{-h}^{0} A(t, s) \phi(s) d s=\int_{-h}^{0} d_{\theta} H(t, \theta) \phi(\theta)\right.$

Where, $0 \leq w_{n} \leq h$, and $\left|\int_{-h}^{0} d_{\theta} H(t, \theta) \phi(\theta)\right| \leq h(\theta)\|\phi\|$.

$D\left(t, x_{t}\right)$ is non - atomic at zero (that is $D\left(t, x_{t}\right)$ is differentiable and integrable at zero).

$$
\int_{-h}^{0} A(t, s) d s+\sum_{n-1}^{\infty}|A(t)| \geq \delta(\varepsilon), \text { for all } t, \text { where } \delta(\varepsilon) \rightarrow 0,
$$

$f$ is continuous and satisfies other smoothness conditions. Klamka(1980)

\subsection{Linearization}

We can linearize the system (1.1) as described by Chukwu (1992) by setting $x_{t}=z$ in $L$; a specified function insider the function $L\left(t, x_{t}\right) x_{t}$ to have $L(t, z) x_{t}$ without loss of generality. Thus system (1.1) becomes

$\frac{d}{d t}\left[D\left(t, x_{t}\right)\right]=L(t, z) x_{t}+\int_{0}^{\infty} A(t, \theta) x(t+\theta) d \theta$

Evidently,

$$
\left.+\int_{-h}^{0} d_{\theta} H(t, \theta)\right] u(t+\theta)+f\left(t, x_{t}\right)
$$

$L(t, z) x_{t}=\sum_{k=0}^{\infty} A_{k} x\left(t-w_{k}\right)+\int_{-\infty}^{0} A(t, \theta) x(t+\theta) d \theta+\int_{0}^{\infty} A(t, \theta) x(t+\theta) d \theta$

$L^{*}(t, Z) x_{t}=\sum_{k=0}^{\infty} A_{k} x\left(t-w_{k}\right)+\int_{-\infty}^{0} A(t, \theta) x(t+\theta) d \theta$

The representation $L, L^{*}$ are the same under the following assumptions;

$$
L(t, Z) x_{t}=\operatorname{limit}_{p \rightarrow \infty} \sum_{k=0}^{p} A_{k} x\left(t-w_{k}\right)+\operatorname{limit}_{M . N \rightarrow \infty} \int_{M}^{N} A(t, \theta) x(t+\theta) d \theta .
$$

We assume the limits exist, giving finite partial sum for the infinite series and the improper integrals. Thus the system

$$
L^{*}(t, Z) x_{t}=\sum_{k=0}^{\infty} A_{k} x\left(t-w_{k}\right)+\int_{-\infty}^{0} A(t, \theta) x(t+\theta) d \theta
$$

is finite and well defined function.

In the light of the above, the system (1.1) reduces to

$x\left(t_{0}\right)=\phi \in C$.

$$
\frac{d}{d t}\left[D(t, Z) x_{t}\right]=L(t, z) x_{t}+\int_{-h}^{0}\left[d_{\theta} H(t, \theta) u(t+\theta)+f\left(t, x_{t}\right)\right.
$$




$$
\text { Where, } L(t, Z) x_{t}=\sum_{k=0}^{p} A_{k} x\left(t-w_{k}\right)+\int_{-h}^{0} A(t, \theta) x(t+\theta) d \theta \text {. }
$$

\subsection{Variation of Constant Formula}

Integrating system (1.3), after linearizing, we have

$$
\begin{aligned}
x(t)=X\left(t, t_{0}, \phi, u\right) x_{0} & +\int_{t_{0}}^{t} X(t, s) f\left(s, x_{s}\right) d s \\
& +\int_{0}^{t} X(t, s)\left\{\int_{-h}^{0} d_{\theta} H(t, \theta) u(t+\theta)\right\} d s
\end{aligned}
$$

Where $X(t, s)$ is the fundamental matrix of the homogeneous part of the system (1.3). $X(t, s)=1$ (identity matrix of nxn) order, for $s=t$.

The 3 rd term in the right hand side of system(1.4) contains the values of the control $u(t)$ for $t<t_{0}$, as well as for $t>t_{0}$. The values of the control $u(t)$ for $t \in\left[t_{0}-h, t_{0}\right]$ enter into the definition of the initial complete state $z(0)=\left\{x_{0}, u_{t_{0}}\right\}$.

To separate them, the $3 \mathrm{rd}$ term of system (1.4) must be transformed by changing the order of integration. Using the unsymmetric Fubini theorem (see Klanka, (1980), we have the following equalities:

$$
\begin{aligned}
& x(t)=X\left(t, t_{0}, \phi, u\right) x_{0}+\int_{0}^{t} X(t, s) f\left(s, x_{s}\right) d s \\
& +\int_{-h}^{0} d_{H_{\theta}}\left(\int_{t_{o}}^{t} X(t, s) H(s, \theta) u(s+\theta) d s\right) \\
& =X\left(t, t_{0}, \phi, u\right) x_{0}+\int_{t_{0}}^{t} X(t, s) f\left(s, x_{s}\right) d s \\
& +\int_{-h}^{0} d_{H_{\theta}}\left(\int_{t_{0}+\theta}^{t+\theta} X(t, s-\theta) H(s-\theta, \theta) u(s-\theta+\theta) d s\right) \\
& =X\left(t, t_{0}, \phi, u\right) x_{0}+\int_{t_{0}}^{t} X(t, s) f\left(s, x_{s}\right) d s \\
& +\int_{-h}^{0} d_{H_{\theta}}\left(\int_{t_{0}+\theta}^{t+\theta} X(t, s-\theta) H(s-\theta, \theta) u(s) d s\right) \ldots \ldots \\
& =X\left(t, t_{0}, \phi, u\right) x_{0}+\int_{t_{0}}^{t} X(t, s) f\left(s, x_{s}\right) d s+\int_{-h}^{0} d_{H_{\theta}}\left(\int_{t_{0}+\theta}^{t_{0}} X(t, s-\theta) H(s-\theta, \theta) u_{0}(s) d s\right) \\
& +\int_{-h}^{0} d_{H_{\theta}}\left(\int_{t_{0}+\theta}^{t_{0}+\theta} X(t, s-\theta) H(s-\theta, \theta) u(s) d s\right)
\end{aligned}
$$

Where the symbol $d_{H_{\theta}}$ denotes that the integration is in the Lebesque - Stieltjes sense with respect to the variable $\theta$ in the function $H(t, \theta)$.

Let us introduce the following notations: 


$$
\widehat{H}(s, \theta)=\left\{\begin{array}{c}
H(s, \theta), \text { for } s \leq t, \theta \in R \\
0, \quad \text { for } s>t, \theta \in R
\end{array}\right\}
$$

Hence $x(t)$ can be expressed in the following form:

$$
\begin{aligned}
& x(t)=X\left(t, t_{0}, \phi, u\right) x_{0}+\int_{t_{0}}^{t} X(t, s) f\left(s, x_{s}\right) d s \\
& +\int_{-h}^{0} d_{H_{\theta}}\left(\int_{t_{0}+\theta}^{t_{0}} X(t, s-\theta) H(s-\theta, \theta) u_{0}(s) d s\right) \\
& +\int_{-h}^{0} d_{H_{\theta}}\left(\int_{t_{0}}^{t} X(t, s-\theta) \widehat{H}(s-\theta, \theta) u(s) d s\right) \ldots \ldots
\end{aligned}
$$

Using again the unsymmetric Fubini theorem, the equality (1.10) can be rewritten in a more convenient form as follows:

$$
\begin{aligned}
x(t)=X\left(t, t_{0}, \phi, u\right) x_{0}+ & \int_{t_{0}}^{t} X(t, s) f\left(s, x_{s}\right) d s \\
& \quad+\int_{-h}^{0} d_{H_{\theta}}\left(\int_{t_{0}+\theta}^{t_{0}} X(t, s-\theta) H(s-\theta, \theta) u_{0}(s) d s\right) \\
& +\int_{t_{0}}^{t}\left[\int_{-h}^{0} X(t, s-\theta) d_{H_{\theta}} H(s-\theta, \theta)\right] u(s) d s \quad \ldots \ldots \ldots
\end{aligned}
$$

Now let us consider the solution $x(t)$ of system (1.2) for $t=t_{1}$, to getting:

$$
\begin{aligned}
x\left(t_{1}\right)=X\left(t, t_{0}, \phi, u\right) x_{0} & +\int_{t_{0}}^{t_{1}} X(t, s) f\left(s, x_{s}\right) d s \\
& \quad+\int_{-h}^{0} d_{H_{\theta}}\left(\int_{t_{0}+\theta}^{t_{0}} X(t, s-\theta) H(s-\theta, \theta) u_{0}(s) d s\right) \\
& +\int_{t_{0}}^{t_{1}}\left[\int_{-h}^{0} X(t, s-\theta) d_{H_{\theta}} \widehat{H}(s-\theta, \theta)\right] u(s) d s
\end{aligned}
$$

Now, let

$$
\begin{gathered}
\alpha(t)=X\left(t, t_{0}, \phi, u\right) x_{0}+\int_{t_{0}}^{t_{1}} X(t, s) f\left(s, x_{s}\right) d s \\
\beta(t)=\int_{-h}^{0} d_{H_{\theta}}\left(\int_{t_{0}+\theta}^{t_{0}} X(t, s-\theta) H(s-\theta, \theta) u_{0}(s) d s\right) \\
\mu(t)=\int_{t_{o}}^{t_{1}}\left[\int_{-h}^{0} X(t, s-\theta) d_{H_{\theta}} \widehat{H}(s-\theta, \theta)\right] u(s) d s . \ldots \ldots \ldots
\end{gathered}
$$

Substituting (1.13), (1.14) and (1.15) into (1.12) we have

$$
x\left(t_{1}\right)=\alpha\left(t_{1}\right)+\beta\left(t_{1}\right)+\int_{t_{0}}^{t_{1}} \mu\left(t_{1}\right) u(s) d s=\mu\left(t_{1}\right)+\beta(t)+R\left(t_{1}, t_{0}\right) .
$$

\section{Definitions}

2.1 Reachable set (see Onwuatu, (1993)) 
The reachable set of the system (1.2) is given as

$$
R\left(t_{1}, t_{0}\right)=\left\{\int_{t_{o}}^{t_{1}}\left[\int_{-h}^{0} X(t, s-\theta) d_{H_{\theta}} \widehat{H}(s-\theta, \theta)\right] u(s) d s: u \epsilon U ;\left|u_{j}\right| \leq 1\right\}
$$

Where, $U=\left\{u \in L_{2}\left(\left[0, t_{1}\right], E^{m}\right)\right.$ and $j=1,2,3, \ldots, m$

\section{3 Attainable Set (see Onwuatu (1993))}

The attainable set for the system (1.2)is the set of all possible solutions of the system (1.2). visa vis system (1.1). It is denoted by

$$
A\left(t_{1}, t_{0}\right)=\left\{x\left(t, x_{0}, u\right): u \in U\right\}, \quad \text { where } U=\left\{u \in L_{2}\left(\left[0, t_{1}\right], E^{m}\right):\left|u_{j}\right| \leq 1 ; \mathrm{j}=1,2, \ldots, \mathrm{m}\right.
$$

\subsection{Target Set (see Onwuatu, (1993))}

The target set of system (1.1) is given as

$$
\begin{aligned}
G\left(t_{1}, t_{0}\right) & =\left\{x\left(t_{1}, x_{0}, u\right): t_{1} \geq \tau>t_{0} \text { for fixed } \tau \text { and } u \in U\right\} \\
\text { where }, U & =\left\{u \in L_{2}\left(\left[0, t_{1},\right], E^{m}\right):\left|u_{j}\right| \leq 1, \quad j=1,2, \ldots \ldots, m\right\}
\end{aligned}
$$

2. 5 Controllability grammian (see Onwuatu (1993))

The controllability grammian of the system (1.1) is given as

$$
\left.\left.W\left(t_{1}, t_{0}\right)=\int_{t_{0}}^{t_{1}}\left[\int_{-h}^{0} X\left(t_{1}, s-\theta\right) d_{H_{\theta}}\right) \widehat{H}(s-\theta, \theta)\right]\left[\int_{-h}^{0} X\left(t_{1}, s-\theta\right) d_{H_{\theta}}\right) \widehat{H}(s-\theta, \theta)\right]^{T} d s
$$

Where $T$ denotes matrix transpose.

\subsection{Complete state(see Onwuatu, (1993))}

The complete state of system (1.1) is given as

$$
Z(t)=\left\{x(t), u_{t}\right\}, \text { while the initial comeplete state } z\left(t_{0}\right)=\left\{x_{0}, u_{0}\right\} .
$$

2. 7 Properness(see Onwuatu,(1993))

The system (1.1) is proper in $E^{n}$ on $\left[t_{0}, t_{1}\right]$ if $\operatorname{span}\left(t_{1}, t_{0}\right)=E^{n}$. That is if

$$
C^{T}\left[\int_{-h}^{0} X(t, s-\theta) d_{H_{\theta}} \widehat{H}(s-\theta, \theta)\right]=0 \text { a.e. } \Rightarrow C=0, C \in E^{n}, t_{1}>0 .
$$

\subsection{Relative Controllability(see Onwuatu (1993))}

The system (Relative controllable on $\left[t_{0}, t_{1}\right]$ if

$$
A\left(t_{1}, t_{0}\right) \cap G\left(t_{1}, t_{0}\right) \neq \phi, \quad t_{1}>0
$$

\section{Main Results}

Optimality conditions for the Nonlinear Infinitely Space of Neutral Differential systems 
with Distributed Delays in the Control.In these systems, the optimal control problem can best be understood in the context of a game of pursuit, (see Balachandran,Dauer and Ananhdi, (2003)).The emphasis here is the search for a control energy that can steer the state of the system of our interest to the target set(which can be a moving point function or a compact set function ) in a minimum time. In other words, the optimal control problem can be stated as follows:

$$
\text { If } t^{*}=\text { infinum }\left\{t: A\left(t_{1}, t_{0}\right) \cap G\left(t_{1}, t_{0}\right) \neq \phi \text { for } t \in\left[t_{0}, t_{1}\right], t>0\right\} \text {. }
$$

Then there exists an admissible control $u^{*}$ such that the solution of the system with this admissible control is steered to the target. The proposition that follows illustrates this assertion.

\section{Proposition 3.1}

Consider the system (1.1)

$$
\frac{d}{d t}\left(D\left(t, x_{t}\right)\right)=L(t, z) x_{t}+\int_{0}^{\infty} A(t, \theta) x(t+\theta) d \theta+\int_{-h}^{0} d \theta H(t, \theta) u(t+\theta)+f\left(t, x_{t}\right)
$$

as a differential game of pursuit, with its basic assumptions.

Suppose that $A\left(t_{1}, t_{0}\right)$ and $G\left(t_{1}, t_{0}\right)$ are compact set functions, then there exists an admissible control $u$ such that the state of the weapon for the pursuit of the target satisfies the system (1.1) if and only if $A\left(t_{1}, t_{0}\right) \cap G\left(t_{1}, t_{0}\right) \neq \phi$.

\section{Proof}

Let $\left\{u^{n}\right\}$ be a sequence of controls in $U$. Since the constraint control $U$ is compact, then the sequence $\left\{u^{n}\right\}$ has a limit $u$, as in tends to infinity. That is

$$
\operatorname{limit}_{n \rightarrow \infty} u^{n}=u
$$

Suppose that the state $y(t)$ of the weapon for the pursuit of the target satisfies the system (1.1) on the time interval $\left[t_{0}, t_{1}\right]$, then $y(t) \in G(t, 0)$, for $t \in\left[t_{0}, t_{1}\right]$.

We are to show that there exists solution $x(t, u) \in A\left(t_{1}, t_{0}\right)$, for $t \in\left[t_{0}, t_{1}\right]$ such that $y(t)=x(t, u)$ for some $u \in U . N o w, x(t, u) \in A(t, 0)$ and from

$$
\begin{aligned}
x\left(t_{1}, x_{0}, u^{n}\right)=X\left(t_{1}, t_{0}, \phi\right) & x_{0}+\int_{t_{0}}^{t_{1}} X(t, s) f\left(s, x_{s}\right) d s \\
& \quad+\int_{-h}^{0} d_{H_{\theta}}\left(\int_{t_{0}+\theta}^{t_{0}} X\left(t_{1}, s-\theta\right) H(s-\theta, \theta) u_{0}^{n}(s) d s\right) \\
& +\int_{t_{o}}^{t_{1}}\left[\int_{-h}^{0} X\left(t_{1}, s-\theta\right) d_{H_{\theta}} \widehat{H}(s-\theta, \theta)\right] u^{n}(s) d s \ldots \ldots . .
\end{aligned}
$$

Taking limit on both sides of the system (3.2), We have

$$
\operatorname{limit}_{n \rightarrow \infty} x\left(t_{1}, x_{0}, u^{n}\right)=\operatorname{limit}_{n \rightarrow \infty} X\left(t_{1}, x_{0}, u^{n}\right) x_{0}
$$




$$
\begin{gathered}
+\int_{t_{0}}^{t_{1}} X(t, s) f\left(s, x_{s}\right) d s+\int_{-h}^{0} d_{H_{\theta}}\left(\int_{t_{0}+\theta}^{t_{0}} X\left(t_{1}, s-\theta\right) H(s-\theta, \theta) \operatorname{limit}_{n \rightarrow \infty} u_{0}^{n}(s) d s\right) \\
+\int_{t_{0}}^{t_{1}}\left[\int_{-h}^{0} X\left(t_{1}, s-\theta\right) d_{H_{\theta}} \widehat{H}(s-\theta, \theta)\right] \operatorname{limit}_{n \rightarrow \infty} u^{n}(s) d s, \text { for } t \in\left[t_{0}, t_{1}\right] \\
\Rightarrow x\left(t_{1}, x_{0}, u\right)=X\left(t_{1}, t_{0}, \phi, u\right) x_{0}+\int_{t_{0}}^{t_{1}} X(t, s) f\left(s, x_{s}\right) d s \\
+\int_{-h}^{0} d_{H_{\theta}}\left(\int_{t_{0}+\theta}^{t_{0}} X\left(t_{1}, s-\theta\right) H(s-\theta, \theta) u_{0}(s) d s\right) \\
+\int_{t_{0}}^{t_{1}}\left[\int_{-h}^{0} X\left(t_{1}, s-\theta\right) d_{H_{\theta}} \widehat{H}(s-\theta, \theta)\right] u(s) d s \\
=x\left(t_{1}, x_{0}, u\right) \in A\left(t_{1}, t_{0}\right) .
\end{gathered}
$$

Since $A\left(t_{1}, t_{0}\right)$ is compact and $\operatorname{limit}_{n \rightarrow \infty} x\left(t_{1}, x_{0}, u^{n}\right)=x\left(t_{1}, x_{0}, u\right)$, there exists a control $u \in U$, such that $x\left(t_{1}, x_{0}, u\right)=y\left(t_{1}\right)$, for $t>0$.

Since $y\left(t_{1}\right) \in G\left(t_{1}, t_{0}\right)$ and also is in $A\left(t_{1}, t_{0}\right)$, it follows that

$$
A\left(t_{1}, t_{0}\right) \cap G\left(t_{1}, t_{0}\right) \neq \phi \text {, for } t \in\left[t_{0}, t_{1}\right]
$$

Conversely,

Suppose that the intersection condition holds; $A(t, 0) \cap G(t, 0) \neq \phi, t \in\left[t_{0}, t_{1}\right]$, then there exists $y(t) \in A\left(t_{1}, t_{0}\right)$ such that $y(t) \in G\left(t_{1}, t_{0}\right), t_{1}>t_{0}$

This implies that $y(t)=x\left(t_{1}, x_{0}, u\right)$ and hence establishes that the state of the weapon of pursuit of the target satisfies the system (1.1). This completes the proof.

Theorem 3.1 (sufficient conditions for the existence of an optimal control).

Suppose that the system (1.1), that is

$$
\begin{aligned}
\frac{d}{d t}\left(D\left(t, x_{t}\right)\right)=L\left(L(t, z) x_{t}+\int_{0}^{\infty}\right. & A(t, \theta) x(t+\theta) d \theta \\
& +\int_{-h}^{0} d \theta H(t, \theta) u(t+\theta)+f\left(t, x_{t}\right)
\end{aligned}
$$

$x\left(t_{0}\right)=\phi$,

is relatively controllable on the finite interval $\left.t_{0}, t_{1}\right]$, then there exists an optimal control.

\section{Proof}

By the controllability of the system (1.1), the intersection condition holds. That is,

$$
A\left(t_{1}, t_{0}\right) \cap G\left(t_{1}, t_{0}\right) \neq \phi .
$$

Also as $x\left(t_{1}, x_{0}, u\right) \in G\left(t_{1}, t_{0}\right)$, so $y\left(t_{1}\right)=x\left(t_{1}, x_{0}, u\right)$.

Recall that the attainable set $A\left(t_{1}, t_{o}\right)$ is a translation of the reachable set $R\left(t_{1}, t_{o}\right)$ 
through the origin $\mathrm{\eta}$ which is given as:

$$
\begin{aligned}
& \eta=X\left(t_{1}, t_{0}, \phi, u\right) x_{0}+\int_{t_{0}}^{t_{1}} X(t, s) f\left(s, x_{s}\right) d s \\
& +\int_{-h}^{0} d_{H_{\theta}}\left(\int_{t_{0}+\theta}^{t_{0}} X\left(t_{1}, s-\theta\right) H(s-\theta, \theta) u_{0}(s) d s .\right.
\end{aligned}
$$

It follows that $y(t) \in A\left(t_{1}, t_{0}\right)$ for $t \in\left[t_{0}, t_{1}\right], t>0$ and can be defined as

$$
y(t)=\int_{t_{0}}^{t}\left[\int_{-h}^{0} X\left(t_{1}, s-\theta\right) d_{H_{\theta}} \widehat{H}(s-\theta, \theta)\right] u(s) d s .
$$

Let $t^{*}=$ infimum $\left\{t: y(t) \in R\left(t_{1}, t_{0}\right), t \in\left[t_{0}, t_{1}\right]\right\}$

Now, $\quad t>0$ and there is a sequence of times $\left\{t_{n}\right\}$ and the corresponding sequence of controls $\left\{t_{n}\right\} \in U$ with $\left\{t_{n}\right\}$ converging to $t^{*}$ (the minimum time).

Let $y\left(t_{n}\right)=z\left(t_{n}\right)=z\left(t_{n}, u^{n}\right)$

Let $y\left(t_{n}\right)=z\left(t_{n}, u^{n}\right) \in R\left(t_{1}, t_{0}\right)$.

Also

$$
\begin{gathered}
\left|y\left(t^{*}\right)-z\left(t^{*}, u^{n}\right)\right|=\left|y\left(t^{*}\right)-y\left(t_{n}\right)+y\left(t_{n}\right)-z\left(t^{*}, u^{n}\right)\right| \\
\leq\left|y\left(t^{*}\right)-y\left(t_{n}\right)\right|+\left|y\left(t_{n}\right)-z\left(t^{*}, u^{n}\right)\right| \\
\leq\left|y\left(t^{*}\right)-y\left(t_{n}\right)\right|+\left|z\left(t_{n}, u^{n}\right)-z\left(t^{*}, u^{n}\right)\right| \\
\leq\left|y\left(t^{*}\right)-y\left(t_{n}\right)\right|+\int_{t^{*}}^{t_{n}}|| z(s)|| d s
\end{gathered}
$$

By the continuity of $y(t)$ which follows the continuity of $R\left(t_{1}, t_{0}\right)$ as a continuous set function and the integrability of $\|z(t)\|$, it follows that $z\left(t^{*}, u^{n}\right) \rightarrow y\left(t^{*}\right)$ as $n \rightarrow \infty$, where $y\left(t^{*}\right)=z\left(t^{*}, u^{*}\right) \in R\left(t_{1}, t_{0}\right)$.

For some $u^{*} \in U$ and by de finition of $t^{*}, u^{*}$ is an optimal control.

This establishes the existence of an optimal control for the nonlinear infinitely space of Neutral Functional Differential System with distributed delays in the control system (1.2) /system(1.1).

\section{Remark 3. 1}

The above stated and proved proposition in other words states that in any game of pursuit described by a nonlinear infinitely space of Neutral functional Differential Systems with distributed delays in control, it is always possible to obtain the control energy function to steer the system state to the target in finite time. The next theorem is, therefore, a consequence of this understanding and provides sufficient conditions for the existence of the control that is capable of steering the state of the system (1.2)visa vis system (1.1) to the target set in minimum time.

\section{Examples}

\section{Example 4.1}

Consider the motion of Satellite in a central gravitational field. Let the kinetic energy $T$ and the potential energy $P$ be given by 


$$
T=\frac{1}{2} m\left(\dot{r}^{2}, r^{2} \dot{\varphi}^{2}\right), P=\frac{y m}{r}
$$

where, $m$ is the mass of the satellite, if the product of the mass of the planet and the gravitational constant $r$ and $\varphi$ are polar coordinate of the satellite (which we idealized as a particle).

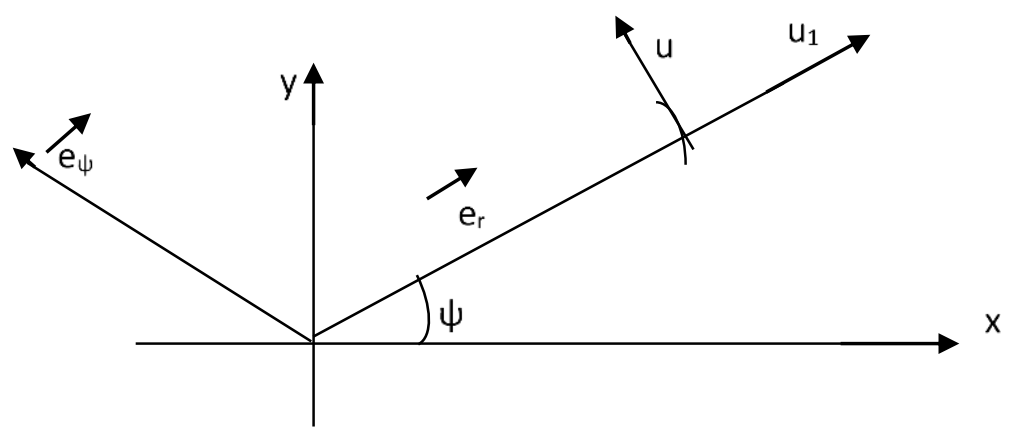

The equations of motion are then

$$
\begin{gathered}
\ddot{r}=\frac{\eta}{r^{2}}+r \dot{\varphi}^{2} \\
\ddot{\varphi}=\frac{-2 \dot{\varphi} \dot{r}}{r}
\end{gathered}
$$

Particular solution is given by the motion in a circular orbit with

$$
r=R, \dot{\varphi}=\omega=\sqrt{\eta \frac{1}{R^{3}}}
$$

Deviations from this orbit are to be corrected by two rocket engines with thrust vectors in the direction $e_{r}$ and $e_{\varphi}$ respectively. Thus, the equation of motion now becomes

$$
\begin{array}{r}
\ddot{r}=\frac{-\eta}{r^{2}}+r \dot{\varphi}^{2}+u_{1} \\
\ddot{\varphi}=\frac{-2 \dot{\varphi} \dot{r}}{r}+\frac{u_{2}}{r}
\end{array}
$$

where $u_{1}$ and $u_{2}$ are the radial and transverse (normal)components of the acceleration respectively (with respect to the circular orbit) as produced by the rocket engines.

Let us now introduce the following new variables:

$$
\begin{gathered}
x_{1}=r-R \\
x_{2}=\dot{x}_{1}=\dot{r} \\
x_{3}=(\varphi-\omega t) R \\
x_{4}=\dot{x}_{3}=(\dot{\varphi}-\omega) R
\end{gathered}
$$

which represent the (small) deviations from the ideal motion. Now,

$$
\begin{aligned}
& \dot{x}_{2}=\ddot{x}_{1}=\ddot{r}=\dot{r} \varphi^{2}-\frac{\eta}{r^{2}}+u_{1} \\
& =\left(x_{1}+R\right) \omega^{2}-\frac{\omega^{2} R^{3}}{\left(x_{1}+R\right)^{2}}+u_{1} \\
& =\left(x_{1}+R\right) \omega^{2}-R \omega^{2}\left(1+\frac{x_{1}}{R}\right)^{-2}+u_{1} \\
& =\left(x_{1}+R\right) \omega^{2}-R \omega^{2}\left[1-2\left(\frac{x_{1}}{R}\right)+3\left(\frac{x_{1}}{R}\right)^{2}-4\left(\frac{x_{1}}{R}\right)^{3}+\ldots\right]+ \\
& =3 \omega^{2} x_{1}-3 \omega^{2} x_{1}{ }^{2} R^{-1}+4 \omega^{2} x_{1}{ }^{3} R^{-2}-
\end{aligned}
$$

$u_{1}$

$5 \omega^{2} x_{1}^{4} 4 R^{-3}+\ldots+u_{1}$ (using Binomial Theorem).

$\Rightarrow \quad \dot{x}_{2}=3 \omega^{2} x_{1}+u_{1}+$ h.o.t (higher order terms)

$$
\dot{x}_{4}=\ddot{x}_{3}=\ddot{\varphi} R=\left(-2 \omega \dot{r} \frac{1}{r}+\frac{u_{2}}{r}\right) R=-2 \omega R x_{2}\left(x_{1}+R\right)^{-1}+u_{2} R\left(x_{1}+R\right)^{-1}
$$


$\ldots\}$

$$
\begin{gathered}
=-2 \omega x_{2}\left(1+\frac{x_{1}}{R}\right)^{-1}+u_{2}\left(1+\frac{x_{1}}{R}\right)^{-1} \\
=\left(-2 \omega x_{2}+u_{2}\right)\left(1+\frac{x_{1}}{R}\right)^{-1} \\
=\left(-2 \omega x_{2}+u_{2}\right)\left\{1-\left(\frac{x_{1}}{R}\right)^{1}+\left(\frac{x_{1}}{R}\right)^{2}-\left(\frac{x_{1}}{R}\right)^{3}+\right. \\
=-2 \omega x_{2}+u_{2}+\text { h.o.t. }
\end{gathered}
$$

Thus, the linearized system yields

$$
\begin{aligned}
& \dot{x}_{1}=x_{2} \\
& \dot{x}_{2}=3 \omega^{2} x_{1}+u_{1} \\
& \dot{x}_{3}=x_{4} \\
& \dot{x}_{4}=-2 \omega x_{2}+u_{2}
\end{aligned}
$$

with $\bar{x}^{T}=\left(\begin{array}{llll}x_{1} & x_{2} & x_{3} & x_{4}\end{array}\right)$, and $\bar{u}^{T}=\left(u_{1} u_{2}\right)$, We have the ordinary linear control system of the form:

$$
\begin{aligned}
& \dot{x}=A \dot{(t}) x(t)+B(t) u(t)=\left(\begin{array}{cccc}
0 & 1 & 0 & 0 \\
3 \omega^{2} & 0 & 0 & 0 \\
0 & 0 & 0 & 1 \\
0 & -2 \omega & 0 & 0
\end{array}\right)\left(\begin{array}{l}
x_{1} \\
x_{2} \\
x_{3} \\
x_{4}
\end{array}\right)+\left(\begin{array}{ll}
0 & 0 \\
1 & 0 \\
0 & 0 \\
0 & 1
\end{array}\right)\left(\begin{array}{l}
u_{1} \\
u_{2} \\
u_{3} \\
u_{4}
\end{array}\right) \text { with } \\
& A=\left[\begin{array}{cccc}
0 & 1 & 0 & 0 \\
3 \omega^{2} & 0 & 0 & 0 \\
0 & 0 & 0 & 1 \\
0 & -2 \omega & 0 & 0
\end{array}\right] \text { and } B=\left[\begin{array}{ll}
0 & 0 \\
1 & 0 \\
0 & 0 \\
0 & 1
\end{array}\right]
\end{aligned}
$$

which provides a first approximation to the controlled motion in a neighborhood of the reference orbit.To determine the controllability of the system, we compute

$$
\operatorname{rank}\left(B, A B, A^{2} B, A^{3} B\right)=n<\infty
$$

and show that it equals 4.

Now, $\operatorname{rank}\left(B, A B, A^{2} B, A^{3} B\right)=$

$\left(\begin{array}{ll}0 & 0 \\ 1 & 0 \\ 0 & 0 \\ 0 & 1\end{array}\right),\left(\begin{array}{cccc}0 & 1 & 0 & 0 \\ 3 \omega^{2} & 0 & 0 & 0 \\ 0 & 0 & 0 & 1 \\ 0 & -2 \omega & 0 & 0\end{array}\right) \cdot\left(\begin{array}{ll}0 & 0 \\ 1 & 0 \\ 0 & 0 \\ 0 & 1\end{array}\right),\left(\begin{array}{cccc}0 & 1 & 0 & 0 \\ 3 \omega^{2} & 0 & 0 & 0 \\ 0 & 0 & 0 & 1 \\ 0 & -2 \omega & 0 & 0\end{array}\right)^{2}\left(\begin{array}{ll}0 & 0 \\ 1 & 0 \\ 0 & 0 \\ 0 & 1\end{array}\right),\left(\begin{array}{cccc}0 & 1 & 0 & 0 \\ 3 \omega^{2} & 0 & 0 & 0 \\ 0 & 0 & 0 & 1 \\ 0 & -2 \omega & 0 & 0\end{array}\right)^{3}\left(\begin{array}{ll}0 & 0 \\ 1 & 0 \\ 0 & 0 \\ 0 & 1\end{array}\right)$

$=\operatorname{Rank}\left[\begin{array}{cccccccc}0 & 0 & 1 & 0 & 0 & 0 & 3 \omega^{2} & 0 \\ 1 & 0 & 0 & 0 & 3 \omega^{2} & 0 & 0 & 0 \\ 0 & 0 & 0 & 1 & -2 \omega & 0 & 0 & 0 \\ 0 & 1 & -2 \omega & 0 & -6 \omega^{3} & 0 & 0 & 0\end{array}\right]=4=n$.

Thus, the system is completely controllable. This means that the satellite can be steered to "always" move in the circular orbit.

Suppose one of the rocket engines breaks down or is shut down for some reason; will the system remain completely controllable? Let us suppose the engine providing the radial thrust is shut down, then only the transverse (tangential) thrust is available, the $B^{T}=\left(\begin{array}{llll}0 & 0 & 0 & 1\end{array}\right)$.

Computing, we have $\operatorname{Rank}\left(B, A B, A^{2} B, A^{3} B\right)=\operatorname{rank}\left[\begin{array}{llll}0 & 0 & 0 & 0 \\ 0 & 0 & 0 & 0 \\ 0 & 1 & 0 & 0 \\ 1 & 0 & 0 & 0\end{array}\right]=2 \neq 4$

The system, therefore, is no longer completely controllable.

Suppose now the engine providing the transverse thrust is shut down, then only the radial thrust is 
available. Then $B^{T}=\left(\begin{array}{llll}0 & 1 & 0 & 0\end{array}\right)$

Computing, we have $\operatorname{Rank}\left(B, A B, A^{2} B, A^{3} B\right)=\operatorname{rank}\left[\begin{array}{cccc}0 & 1 & 0 & 3 \omega^{2} \\ 1 & 0 & 3 \omega^{2} & 0 \\ 0 & 0 & -2 \omega & 0 \\ 0 & -2 \omega & 0 & -6 \omega^{3}\end{array}\right]=3 \neq 4$

The system is again no longer completely controllable.Thus, once one of the engines is shut down, the satellite can no longer be "constrained" to move along the circular (reference) orbit.

\section{Example 4.2}

Consider the system

$\frac{d}{d t}\left(x(t)-A_{-1} x(t-h)\right)=A_{0} x(t)+A_{1} x(t-h)+B u(t)+e^{-a t} \sin (x(t)+x(t-h)) \cos u$

where $, A_{I}, i=0, \pm 1$ are constant matrices given by: $A_{-1}=\left(\begin{array}{cc}0 & \frac{1}{4} \\ \frac{1}{4} & 0\end{array}\right)$,

$A_{0}=\left(\begin{array}{cc}-3 & 1 \\ 3 & -4\end{array}\right), \quad A_{1}=\left(\begin{array}{cc}0 & \frac{3}{4} \\ 0 & \frac{-1}{4}\end{array}\right), B=\left(\begin{array}{l}0 \\ 1\end{array}\right)$ and $f=\left(e^{-a t} \sin (x(t)+x(t-h)) \cos u\right)$.

We now show that the linear base system

$$
\frac{d}{d t}\left(x(t)-A_{-1} x(t-h)\right)=A_{0} x(t)+A_{1} x(t-h)+B u(t) \ldots \ldots \ldots \ldots \ldots \ldots
$$

is relatively controllable or proper.

We recall that the controllability of a system implies properness of the system.

Thus we need to show that,

$$
\operatorname{rank}\left[B, A_{0} B\right]=n<\infty \text {. (by R.E.Kalman controllability criterion) }
$$

But $\operatorname{rank}\left[B, A_{0} B\right]=\operatorname{rank}\left(\begin{array}{cc}0 & 1 \\ 1 & -4\end{array}\right)=\operatorname{rank}\left(\begin{array}{ll}1 & 1 \\ 1 & 0\end{array}\right)=2=n<\infty$.

Therefore, the system(4.2) is proper,

Moreover, by th invocation of Onwuatu, (1993), if asystem is relatively controllable , there exists an optimal control which unique and Bang - bang.

Thus the system (4.2) has an optimal control since properness of any dynamical system implies its relative controllabilty (see Oraekie, (2015))

\section{Conclusion}

Necessary and Sufficient Conditions for the existence of an optimal control of the perturbed nonlinear systems with distributed delays in the control have been established.These conditions are given with respect to: (a) the establishment of the fact that in these systems,

the optimal control problem can best be understood in the context of a game of pursuit.

The emphasis here is the search for a control energy function that can steer the state of the system of our interest to the target set in a minimum time. (b) The system being relatively controllable with the assumption that the perturbation $f$ satisfies some smooth and growth conditions. Computable criteria for all these are reported and examples are given. These results extended known results in the literature.

\section{References}

Artstein, Z and Tradmar, G. (1982). "Linear Systems with Indirect Controls - the Underlying measure”, S.I.A.M., Journal on control and optimization, 20: $96-111$. 
Balachandran, K. and Anandhi, E.R. (2003), "Neutral Functional Integrodifferential control Systems in Banach Spaces", Kybernetika 39(3): $359-367$.

Balanchandran, K. and Dauer, J.P (1989), "Relative Controllability of Perturbation of Nonlinear Systems", Journal of Optimization Theory and Applications, 63: 51 - 56.

Chukwu, E.N. (1992). "Stability and Time Optimal Control of Hereditary Systems,” Academic Press, New York.

Chukwu, E.N. (2001). "Differential Models and Neutral Systems for Controlling the Wealth of Nations" Services on Advance in Mathematics from Applied Sciences, Vol.54. World Scientific, New Jersey.

Fu, (2004), "Controllability of Abstract Neutral Functional Differential Systems with unbounded delay", Applied Mathematics and Computation 151(2) 299 - 314.

Klanka, J. (1976), Relatively Controllability and Minimum Energy Control of Linear Systems with Distributed Delay in Control”, E.E.E. Transactions in Automatic Control -21, Pp 594 - 595.

Klanka, J. (1980). "Controllablity of Nonlinear Systems with Distributed Delay in Control”, Int. Journal of Control, $31(5), 811$ $-819$.

Onwuatu, J.U. (1984), "On the Null Controllability in function space of Nonlinear Systems of Neutral Functional Differential Equations with Limited Controls”. Journal of Optimization Theory and Applications 42: 397 - 420.

Onwuatu, J.U. (1993). "Null Controllability of Nonlinear Infinite Neutral Systems”, Kyberuetika, 29: 1-12.

Oraekie, P.A. (2015). Relative Controllability of Neutral Functional Integrodifferential Systems in Banach spaces with Distributed Delays in the Control, American Academic and Scholarly Research Journal, 7(5), 115-123.

\section{$\underline{\text { How to cite this article }}$}

Oraekie, P.A. (2018). Optimal Controllability of Nonlinear Infinitely Space of Neutral Differential Systems with Distributed Delays in the Control. Tropical Journal of Applied Natural Sciences, 2(2): 42-54. Doi: https://doi.org/10.25240/TJANS.2018.2.2.06. 